\title{
Tsafon
}

Revue d'études juives du Nord

$80 \mid 2020$

Varia

\section{Narration, transmission et traumatisme psychique}

Une lecture de l'essai Le conteur de Walter Benjamin

\section{Olivier Taïeb}

\section{(2) OpenEdition}

\section{Journals}

Édition électronique

URL : https://journals.openedition.org/tsafon/3195

DOI : $10.4000 /$ tsafon.3195

ISSN : 2609-6420

Éditeur

Association Jean-Marie Delmaire

\section{Édition imprimée}

Date de publication : 1 décembre 2020

Pagination : 67-82

ISSN : $1149-6630$

\section{Référence électronique}

Olivier Taïeb, "Narration, transmission et traumatisme psychique», Tsafon [En ligne], 80 | 2020, mis en ligne le 01 décembre 2020, consulté le 24 juin 2021. URL : http://journals.openedition.org/tsafon/3195 ; DOI : https://doi.org/10.4000/tsafon.3195 


\title{
Narration, transmission et traumatisme psychique
}

\section{Une lecture de l'essai Le conteur de Walter Benjamin}

\author{
Olivier Taïeb*
}

L'essai Le conteur ${ }^{1}$ de Walter Benjamin a été publié en 1936, trois ans après son texte Expérience et pauvreté ${ }^{2}$ dont une partie est reprise littéralement dans la section I de l'essai. Benjamin fait le constat d'une « chute du cours de l'expérience », en particulier après la guerre de 19141918, entraînant une crise du récit et une crise de la transmission. La résonance de cet essai avec les écrits psychanalytiques sur le traumatisme psychique est évidente même si Benjamin n'y cite pas explicitement Freud, alors qu'il s'appuie, par exemple, sur Au-delà du principe de plaisir dans ses écrits sur Baudelaire ${ }^{3}$ publiés en $1939^{4}$. Ses Thèses sur

\footnotetext{
* Praticien hospitalier, HDR, Service de Psychiatrie, Hôpital Avicenne, APHP, Université Paris 13, Bobigny et CESP Inserm U1018, Villejuif.

${ }^{1}$ Walter Benjamin, « Le conteur. Réflexions sur l'œuvre de Nicolas Leskov », traduit de l'allemand par Maurice de Gandillac revu par Pierre Rusch, dans Euvres III, Paris, Gallimard, coll. Folio Essais, 2000 [1936], p. 114-151.

${ }^{2}$ Walter Benjamin, « Expérience et pauvreté », traduit de l'allemand par Pierre Rusch, dans Euvres II, Paris, Gallimard, coll. Folio Essais, 2000 [1933], p. 364-372.

${ }^{3}$ Walter Benjamin, «Sur quelques thèmes baudelairiens », traduit de l'allemand par Maurice de Gandillac revu par Rainer Rochlitz, dans Euvres III, Paris, Gallimard, coll. Folio Essais, 2000 [1939], p. 329-390.

${ }^{4}$ Dans son journal en 1930, Benjamin envisageait déjà la relation entre Proust et Freud, sur laquelle il reviendra dans son essai sur Baudelaire : "J'admets que Proust, au fond, peut-être se range du côté de la mort. Au-delà du principe du plaisir, l'œuvre géniale de la vieillesse de Freud, est probablement le commentaire fondamental de l'œuvre de Proust » (Walter Benjamin, « Journal parisien », dans Sur Proust, traduit de l'allemand par Robert Kahn, Caen, Nous, 2015 [textes rédigés en 1930 selon les éditeurs de ses œuvres], p. 114.).
} 
l'histoire ${ }^{5}$ présentent, elles aussi, les signes d'une lecture attentive de Freud $^{6}$.

Cet article propose d'expliciter cette part « cachée » de l'essai de Benjamin et de montrer en retour comment il peut contribuer à éclairer les implications politiques de la psychanalyse.

\section{L'essai Le conteur. Réflexions sur l'œuvre de Nicolas Leskov}

L'essai Le conteur. Réflexions sur l'œuvre de Nicolas Leskov a été publié dans la revue suisse Orient und Okzident en octobre 1936. Une traduction française par Benjamin lui-même est parue de façon posthume en 1952 sous le titre Le Narrateur ${ }^{7}$. Maurice de Gandillac et Pierre Rusch ont choisi le terme «conteur» pour traduire l'allemand Erzähler, le narrateur désignant le plus souvent une figure interne au discours. Dans la même perspective, le terme «raconteur» a été préféré dans des traductions récentes ${ }^{8}$. Même si ce texte n'est pas une monographie sur l'œuvre de Nicolas Leskov, les caractéristiques de certains de ses récits illustrent parfaitement les propos de Benjamin. Leskov est un écrivain russe qui est né près de la ville d'Oriol en 1831 et qui est mort en 1895 à Saint-Pétersbourg. Pour Benjamin, «l'activité qui fut sans doute la plus profitable à son travail littéraire est celle qu'il exerça longtemps comme représentant pour la Russie d'une firme anglaise. À ce titre, il eut l'occasion de voyager à travers tout le pays ${ }^{9}$.

Leskov a donné, en effet, à certains de ses récits la forme du skaz ou dit qui est un récit à deux voix, celle du conteur et celle de l'auteur ${ }^{10}$. Le skaz tente un retour aux formes archaïques du récit :

\footnotetext{
${ }^{5}$ Walter Benjamin, « Sur le concept d'histoire », Écrits français, Paris, Gallimard, coll. Folio Essais, 2003 [textes rédigés en 1940 selon les éditeurs de ses œuvres], p. 423-455. ${ }^{6}$ Barbara Kleiner, «L'éveil comme catégorie centrale de l'expérience historique dans le Passagen-Werk de Benjamin », dans Heinz Wismann (dir.), Walter Benjamin et Paris, Paris, Les éditions du Cerf, 1986, p. 497-515.

7 Walter Benjamin, «Le Narrateur. Réflexions à propos de l'œuvre de Nicolas Leskov », dans Écrits français, Paris, Gallimard, coll. Folio Essais, 2003 [textes rédigés en 1936 selon les éditeurs de ses œuvres], p. 264-298.

${ }^{8}$ Walter Benjamin, « Le raconteur. Réflexions sur l'œuvre de Nikolaï Leskov », traduit de l'allemand par Maël Renouard, dans Nikolaï Leskov, Le voyageur enchanté, Paris, Payot et Rivages, 2011 [1936], p. 11-48.

Walter Benjamin, Le raconteur. A propos de l'auvre de Nicolas Leskov, traduit de l'allemand par Sibylle Muller, Strasbourg, Circé, 2014 [1936].

${ }^{9}$ W. Benjamin, « Le conteur », op. cit., p. 118.

${ }^{10}$ Catherine Géry, « Introduction », dans Nicolas Leskov, La Lady Macbeth de Mtsensk, traduit du russe par Catherine Géry, Paris, Garnier, 2017, p. 7-54.
} 
[...] mais aussi à un ensemble de pratiques anciennes ou non occidentales lorsque la vive voix (ou la voix vive) était première dans la transmission non seulement du folklore, la tradition orale, mais aussi du texte : dans le passé, on lisait à voix haute; aussi la voix, mais également l'ouie, jouaient un rôle prépondérant. ${ }^{11}$

Le skaz s'adresse à un auditoire fictif mais présent dans le récit. Il renouvelle sans cesse le lien qu'il tisse entre le conteur et son public. Comme le dit Benjamin ${ }^{12}$ : «Celui qui écoute une histoire se trouve en compagnie du conteur ; même celui qui la lit partage cette compagnie ». C'est la fameuse illusion du skaz où la littérature fait semblant d'être encore, à un moment de domination absolue du roman, une pratique collective $^{13}$.

L'essai Le conteur comporte 19 sections. Les sections I, IV, VIII et XIII sont les plus contributives au propos de cet article.

\section{La chute du cours de l'expérience et le traumatisme psychique}

Dans la section I, Benjamin aborde la question historique et politique de la transmission, c'est-à-dire de ce qui fait communauté pour les hommes :

L'art de conter est en train de se perdre. Il est de plus en plus rare de rencontrer des gens qui sachent raconter une histoire. [...] C'est comme si nous avions été privés d'une faculté qui nous semblait inaliénable, la plus assurée entre toutes: la faculté d'échanger des expériences. L'une des raisons de ce phénomène saute aux yeux : le cours de l'expérience a chuté. ${ }^{14}$

\section{Il reprend un passage d'Expérience et pauvreté :}

L'expérience, on savait exactement ce que c'était : toujours les anciens l'avaient apportée aux plus jeunes. [...] Trouve-t-on encore des gens capables de raconter une histoire ? Où les mourants prononcent-ils encore des paroles impérissables, qui se transmettent de génération en génération comme un anneau ancestral ? [...] Une chose est claire : le cours de l'expérience a chuté, et ce dans une génération qui fit en 1914-1918 l'une des expériences les plus effroyables de l'histoire universelle. Le fait, pourtant, n'est peut-être pas si étonnant qu'il n'y paraît. N'a-t-on pas alors constaté que les gens revenaient muets du champ de bataille? Non pas plus riches, mais plus pauvres en expérience communicable.

\footnotetext{
${ }^{11}$ Ibid., p. 34.

${ }^{12}$ W. Benjamin, « Le conteur », op. cit., p. 138.

${ }^{13}$ C. Géry, « Introduction », op. cit.

${ }^{14}$ W. Benjamin, « Le conteur », op. cit., p. 115.
} 
[...] Non, cette dévalorisation n'avait rien d'étonnant. Car jamais expériences acquises n'ont été aussi radicalement démenties que l'expérience stratégique de la guerre de position, l'expérience économique par l'inflation, l'expérience corporelle par l'épreuve de la faim, l'expérience morale par les manœuvres des gouvernants. Une génération qui était encore allée à l'école en tramway hippomobile se retrouvait à découvert dans un paysage où plus rien n'était reconnaissable, hormis les nuages et, au milieu, dans un champ de forces traversé de tensions et d'explosions destructrices, le minuscule et fragile corps humain. ${ }^{15}$

La guerre de 1914-1918 a donc plongé les hommes dans un monde inintelligible, bouleversant leurs repères et leurs expériences antérieures. Ces démentis et cette dévalorisation de leurs expériences ont rompu les liens et la transmission. C'est à cause de l'effondrement du cours de l'expérience que les hommes ne se racontent plus grand-chose et que la figure du conteur s'est éloignée. Si les hommes ne savent plus ou ne peuvent plus se relier entre eux par la transmission, ils ne ressentent alors plus le besoin de raconter des histoires.

C'est la section qui mérite le plus d'être mise en parallèle avec la littérature psychanalytique. Le premier conflit mondial a, en effet, entraîné de très nombreux cas de névroses traumatiques: «La guerre effroyable qui vient juste de terminer son cours a fait naître un grand nombre d'affections de ce type et a du moins mis un terme à la tentation de les ramener à un dommage organique infligé au système nerveux par l'action d'une violence mécanique ${ }^{16}$.

Freud a montré que les réactions psychiques à certaines formes de danger, comme la guerre, se situent au-delà du principe qui régit le plaisir-déplaisir et correspondent alors à des traumatismes auxquels l'organisation psychique n'est pas en mesure de faire face par ses moyens de protection habituels ${ }^{17}$. Il a introduit alors une seconde dualité des pulsions, les pulsions de vie et les pulsions de mort en regard de la première dualité, les pulsions sexuelles et les pulsions d'autoconservation :

Nous supposons que les pulsions de l'homme sont de deux espèces, seulement, soit celles qui veulent conserver et réunir - nous les nommons érotiques, tout à fait dans le sens de l'Eros dans le Banquet de Platon [...] - et d'autres qui

\footnotetext{
${ }^{15}$ W. Benjamin, « Expérience et pauvreté », op. cit., p. 365.

${ }^{16}$ Sigmund Freud, Au-delà du principe de plaisir, traduit de l'allemand par Janine Altounian, André Bourguignon, Pierre Cotet et Alain Rauzy, Paris, PUF, 2010 [1920], p. 10 .

${ }^{17}$ Ibid.
} 
veulent détruire et mettre à mort; nous regroupons celles-ci en pulsion d'agression ou pulsion de destruction. ${ }^{18}$

\section{Ces pulsions sont liées :}

Il semble qu'une pulsion d'une seule espèce ne puisse pour ainsi dire jamais s'exercer isolément, elle est toujours liée - comme nous disons : en un alliage - à un certain moment de l'autre partie, lequel modifie son but ou, dans certaines circonstances, lui permet seul de l'atteindre. ${ }^{19}$

C'est la déliaison pulsionnelle qui est à l'origine des troubles. La compulsion de répétition se substitue alors à la remémoration: «[Le malade] est bien plutôt obligé de répéter le refoulé comme expérience vécue présente, au lieu de s'en souvenir comme un morceau du passé, ce que préfèrerait le médecin ${ }^{20}$. Dès lors l'analyste a pour objectif que le patient parvienne à reconnaître malgré tout ce passé sans cesse actualisé « comme étant encore et toujours un reflet d'un passé oublié »"

Même si le constat final est similaire entre Benjamin et Freud, le processus est différent. Pour Freud, c'est, d'abord, le vécu traumatique de la guerre qui altère les capacités narratives et d'historicisation d'un sujet. L'impact traumatique peut ensuite, bien sûr, se transmettre d'une génération à une autre, en particulier dans les violences collectives.

Pour Benjamin, l'effondrement du cours de l'expérience a rendu les hommes «pauvres" à cause de multiples ruptures avec l'histoire de chaque individu mais aussi avec le patrimoine culturel « de l'humanité entière $»$ :

Cet effroyable déploiement de la technique plongea les hommes dans une pauvreté tout à fait nouvelle. [...] Que vaut en effet tout notre patrimoine culturel, si nous n'y tenons pas, justement par les liens de l'expérience ? [...] Avouons-le: cette expérience ne porte pas seulement sur nos expériences privées, mais aussi sur les expériences de l'humanité entière. Et c'est donc une nouvelle espèce de barbarie. [...] Pauvres, voilà bien ce que nous sommes devenus. Pièce par pièce, nous avons dispersé l'héritage de l'humanité, nous avons dû laisser ce trésor au mont de piété, souvent pour un centième de sa valeur, en échange de la piécette de l' « actuel ${ }^{22}$

\footnotetext{
${ }^{18}$ Sigmund Freud, «Pourquoi la guerre? », dans Actuelles sur la guerre et la mort et autres textes, traduit de l'allemand par Janine Altounian, André Bourguignon, Alice Cherki, Pierre Cotet et Alain Rauzy, Paris, PUF, 2012 [1933], p. 69.

${ }^{19}$ Ibid., p. 70.

${ }^{20}$ S. Freud, Au-delà du principe de plaisir, op. cit., p. 16.

${ }^{21}$ Ibid., p. 17.

${ }^{22}$ W. Benjamin, « Expérience et pauvreté », op. cit., p. 366 et p. 372.
} 
Cette "pauvreté » a aussi été déplorée par Freud dans le texte Actuelles sur la guerre et la mort publié en 1915. Il y dénonce les cruautés et les injustices dont se rendent responsables les États en guerre :

Pris dans le tourbillon de ce temps de guerre [...], nous ne savons même plus quelle signification donner aux impressions qui nous assaillent et quelle valeur accorder aux jugements que nous formons. Il nous semblera que jamais encore un événement n'avait détruit tant de biens précieux communs à l'humanité, frappé de confusion tant d'intelligences parmi les plus claires, si radicalement rabaissé ce qui était élevé. [...] [La guerre] rompt tous les liens faisant des peuples en lutte une communauté et menace de laisser derrière elle une rancœur qui pendant longtemps ne permettra pas de les renouer. ${ }^{23}$

Benjamin, en exil depuis 1933, évoque, bien sûr, la nouvelle guerre qui arrive : "À la porte se tient la crise économique, derrière elle, une ombre, la guerre qui s'apprête $»^{24}$. Cette guerre sera différente de la précédente comme le rappelle Freud dans sa lettre à Albert Einstein en 1932, dans le cadre d'une correspondance organisée par la Société des Nations : «On ne peut pas condamner toutes les espèces de guerre au même degré ; tant qu'il y a des empires et des nations qui sont prêts, sans aucun égard, à en anéantir d'autres, ces autres doivent être armés pour la guerre $»^{25}$.

\section{L'éloignement de la tradition}

Dans la suite de son essai, Benjamin évoque le moment de la chute de l'expérience qui ne remonte pas à la guerre de 1914-1918 mais qui est beaucoup plus ancien :

Le phénomène ne date pas d'aujourd'hui. Et rien ne serait plus insensé que de le considérer comme un simple «signe de décadence », à plus forte raison comme un fait spécifiquement «moderne ». Il s'agit plutôt d'un phénomène concomitant à l'évolution historique des forces productives : ce mouvement qui, au cours des siècles et de façon tout à fait progressive, a éliminé le récit du domaine de la

\footnotetext{
${ }^{23}$ Sigmund Freud, " Actuelles sur la guerre et la mort », dans Actuelles sur la guerre et la mort et autres textes, traduit de l'allemand par Janine Altounian, André Bourguignon, Alice Cherki, Pierre Cotet et Alain Rauzy, Paris, PUF, 2012 [1915], p. 4 et p. 7.

${ }^{24}$ W. Benjamin, « Expérience et pauvreté », op. cit., p. 372.

${ }^{25}$ S. Freud, « Pourquoi la guerre ? », op. cit., p. 74.
} 
parole vivante, a en même temps rendu sensible, dans ce qui ainsi disparaissait, une beauté nouvelle. ${ }^{26}$

En effet, la tradition orale est depuis longtemps devenue plus lointaine et c'est elle qui était, par ses caractéristiques, la plus capable de transmission et de partage :

L'expérience transmise de bouche en bouche est la source à laquelle tous les conteurs ont puisé. Et parmi ceux qui ont couché leurs récits par écrit, les plus grands sont ceux dont le texte s'éloigne le moins de la parole des innombrables conteurs anonymes. $^{27}$

Deux figures du conteur existaient: l'artisan qui avait «la connaissance du passé » et le marin «la connaissance des contrées lointaines $»^{28}$.

C'est l'avènement du roman dont les origines remontent à l'Antiquité mais qui n'a pu vraiment se développer qu'avec l'invention de l'imprimerie, qui a réduit la place du conte. Il a plongé le lecteur dans la solitude, le laissant s'interroger seul sur «le sens de la vie ${ }^{29}$. Si pour le conteur, dont l'image paradigmatique est la Shéhérazade des Mille et une nuits, un récit renvoie à un autre, la fin du roman est définitive et ne laisse pas de place à l'interrogation sur ce qui arriverait après.

Mais l'information dont le règne est plus récent s'oppose au récit comme une forme «beaucoup plus menaçante que le roman » ${ }^{30}$. Elle prétend être «vérifiable »: "Souvent, elle n'est pas plus exacte que ne l'étaient les nouvelles colportées aux siècles passés. Mais alors que ces nouvelles prenaient bien souvent un aspect merveilleux, il est indispensable que l'information paraisse plausible $»^{31}$. Elle ne détient sa valeur que de sa nouveauté : «Elle ne vit qu'en cet instant, elle doit s'abandonner entièrement à lui et s'ouvrir à lui sans perdre de temps. Il n'en est pas de même pour le récit : il ne se livre pas. Il garde sa force rassemblée en lui, et offre, pour longtemps encore, matière à développement $\gg{ }^{32}$.

Giorgio Agamben, dans son ouvrage Enfance et histoire. Essai sur la destruction de l'expérience, reprend le propos de Benjamin. Il ajoute la

\footnotetext{
${ }^{26}$ W. Benjamin, « Le conteur », op. cit., p. 120.

${ }^{27}$ Ibid., p. 116.

${ }^{28}$ Ibid., p. 117

${ }^{29}$ Ibid., p. 137.

${ }^{30}$ Ibid., p. 122.

${ }^{31}$ Ibid., p. 123.

${ }^{32}$ Ibid., p. 124.
} 
science moderne comme une nouvelle cause de la destruction de l'expérience: «La science moderne, contrairement à une opinion souvent reçue, naît d'une méfiance sans précédent envers l'expérience telle que la conçoit la tradition $»^{33}$. L'expérimentation transporte l'expérience «hors de l'homme: dans les instruments et dans les nombres $[\ldots]$. Du même coup, l'expérience traditionnelle perd en réalité toute valeur $»^{34}$.

Il donne un autre exemple de la destruction de l'expérience :

L'actuelle toxicomanie de masse doit être considérée, elle aussi, dans la perspective ouverte par cette destruction de l'expérience. Car entre les nouveaux drogués et les intellectuels qui découvraient la drogue au $\mathrm{XIX}^{\mathrm{e}}$ siècle, la différence est que ceux-ci (en tout cas les moins lucides) pouvaient encore nourrir l'illusion de vivre une expérience inédite, alors qu'il ne s'agit plus pour ceux-là que de se débarrasser de toute expérience. ${ }^{35}$

\section{Crise du récit et crise de la transmission}

Tout récit est adressé à autrui. Sa fonction est d'être au service de la communication et de la transmission. Il apporte à son destinataire un message, une connaissance, une expérience : «Le conteur emprunte la matière de son récit à l'expérience : la sienne ou celle qui lui a été rapportée par autrui. Et ce qu'il raconte, à son tour, devient expérience en ceux qui écoutent son histoire ${ }^{36}$. Pour qu'il y ait transmission, le destinataire, auditeur ou lecteur, doit avoir lui aussi des capacités narratives. Il doit être capable de raconter et de se raconter, c'est-à-dire de mettre en intrigue les différents épisodes de sa vie selon une logique aristotélicienne, explicitée par Paul Ricœur ${ }^{37}$. Pour configurer son propre récit, il faut repérer « des histoires non encore racontées, des histoires qui demandent à être racontées, des histoires qui offrent les points d'ancrage au récit ${ }^{38}$. Comme le dit Benjamin dans Sur quelques thèmes baudelairiens : «Le récit ne se soucie pas de transmettre le pur en-soi de

\footnotetext{
${ }^{33}$ Giorgio Agamben, Enfance et histoire. Destruction de l'expérience et origine de l'histoire, traduit de l'italien par Yves Hersant, Paris, Payot et Rivages, 2010 [1978], p. 30.

${ }_{34}^{34}$ Ibid., p. 30.

${ }^{35}$ Ibid., p. 27.

${ }^{36}$ W. Benjamin, « Le conteur », op. cit., p. 121.

${ }^{37}$ Paul Ricœur, Temps et récit. 1. L'intrigue et le récit historique, Paris, Seuil, coll. Points Essais, 1991 [1983]. Danielle Cohen-Levinas, «Philosopher dans la forme du temps (récit, expérience et révélation) », Europe n¹008, 2013, p. 127-140.

${ }^{38} \mathrm{P}$. Ricœur, Temps et récit. 1, op. cit., p. 141.
} 
l'événement; il l'incorpore à la vie même de celui qui raconte, pour le transmettre, comme sa propre expérience, à ceux qui écoutent. Ainsi le conteur y laisse sa trace, comme la main du potier sur le vase d'argile ${ }^{39}$. L'événement ne relève donc pas de la seule dimension chronologique du récit. Il est aussi intégré dans sa dimension configurante proprement dite. C'est le cercle de la mimèsis qui est plutôt « une spirale sans fin ${ }^{40}$. Le récit est notre façon de vivre dans le monde en le rendant intelligible. Raconter des histoires est aussi le moyen d'accéder à soi et à autrui ${ }^{41}$.

Ce qui peut se transmettre à l'auditeur ou au lecteur peut être un « conseil » :

Le conteur est un homme de bon conseil pour son public. Si l'expression « être de bon conseil» commence aujourd'hui à paraitre désuète, c'est parce que l'expérience devient de moins en moins communicable. [...] Porter conseil, en effet, c'est moins répondre à une question que proposer une manière de poursuivre une histoire (en train de se dérouler). ${ }^{42}$

Les enfants sont, bien sûr, les destinataires privilégiés de ces conseils : "Le conte, qui, aujourd'hui encore, reste le premier conseiller de l'enfance, parce qu'il fut jadis le premier conseiller de l'humanité, se perpétue secrètement dans l'art du récit ${ }^{43}$. Par ses conseils, le conte avait une fonction protectrice : "Quand la détresse était à son comble, il offrait le secours le plus prompt [...]. Il enseigne aujourd'hui encore aux enfants que le plus opportun, pour qui veut faire face aux puissances de l'univers mythique, est de combiner la ruse et l'effronterie $»{ }^{44}$. Mais pour que le destinataire puisse bénéficier de ces conseils, il doit être capable de les raconter à son tour : « Pour pouvoir demander conseil, il faudrait d'abord être capable de raconter cette histoire $\gg{ }^{45}$.

Mais le propre de l'événement traumatique est de ne pas se laisser mettre en intrigue et ainsi de s'opposer au processus d'historicisation. Remémoration et anticipation ne sont plus possibles. Il n'y a plus ni espace d'expérience, ni horizon d'attente. Le passé traumatique ne peut pas se constituer en souvenir et se maintient dans une incessante

\footnotetext{
${ }^{39} \mathrm{~W}$. Benjamin, « Sur quelques thèmes baudelairiens », op. cit., p. 335.

${ }^{40}$ P. Ricour, Temps et récit. 1, op. cit., p. 138.

${ }^{41}$ Paul Ricœur, Soi-même comme un autre, Paris, Seuil, coll. Points Essais, 1996 [1990].

${ }^{42}$ W. Benjamin, « Le conteur », op. cit., p. 119.

${ }^{43}$ Ibid., p. 141.

${ }^{44}$ Ibid., p. 141

${ }^{45}$ Ibid., p. 120.
} 
actualisation. Le récit de soi est menacé et la souffrance devient inénarrable. Le fil narratif est alors rompu par la focalisation sur l'instant. L'instant n'est pas le présent : «L'instant est arraché à la dialectique du triple présent [de saint Augustin], il n'est plus qu'interruption du temps, rupture de la durée; c'est par là que toutes les connexions narratives se trouvent altérées $»^{46}$. Le tissu internarratif intersubjectif peut se déchirer, 1'histoire de chacun étant enchevêtrée dans l'histoire des autres ${ }^{47}$, un tissu qui ressemble au filet de Benjamin. L'enjeu est alors de retisser des liens intra- et intersubjectifs avec ces sujets effractés pour que leurs vies qui n'ont plus de narrateur puissent en quelque sorte en retrouver ${ }^{48}$.

Janine Altounian, essayiste et traductrice de Freud, a beaucoup travaillé sur ce qui se transmet des survivants d'un traumatisme collectif à leurs héritiers. Née à Paris de parents survivants du génocide arménien, elle y a réfléchi à partir de son propre parcours. Benjamin est l'un des auteurs sur lesquels elle s'appuie, en particulier sur le texte Expérience et pauvreté et la section I du Conteur. Le silence de ceux qui reviennent de la guerre de 1914-1918 est rapproché du «manque de parole » entre elle et son père sur son expérience «d'un génocide perpétré justement sur le versant oriental de la guerre de $1914 »^{49}$.

Pour elle, existe un lien manifeste entre la crise de la transmission et l'irruption au début du $\mathrm{XX}^{\mathrm{e}}$ siècle de la violence collective des morts en masse, le plus souvent sans sépulture. La séparation interpsychique des héritiers avec leurs ascendants, vivants ou morts est empêchée ou remplacée par un clivage mutilant défensif pour assurer leurs propres survies psychiques :

Aussi, les processus de transmission étant coextensifs à ceux de la séparation différenciatrice entre individus et entre générations, le travail de deuil qui permettrait d'hériter des disparus et de transmettre leurs traces reste-t-il en suspens et en souffrance de filiation. ${ }^{50}$

\footnotetext{
${ }^{46}$ Paul Ricœur, «La souffrance n'est pas la douleur », dans Jean-Marie von Kaenel (dir.), Souffrances. Corps et âme, épreuves partagées, Paris, Autrement, 1994, p. 63.

${ }^{47}$ P. Ricœur, Soi-même comme un autre, op. cit.,

${ }^{48}$ Paul Ricœur, "La vie : un récit en quête de narrateur », dans Écrits et conférences 1. Autour de la psychanalyse, Paris, Seuil, 2008 [1986], p. 257-276. Myriam Revault d'Allonnes, «La vie refigurée: les implications éthiques du récit», Archives de Philosophie, 74, 2011, p. 599-610.

${ }^{49}$ Janine Altounian, De la cure à l'écriture. L'élaboration d'un héritage traumatique, Paris, PUF, 2012, p. 94.

50 Janine Altounian, «Les héritiers des génocides », dans Françoise Brette, Michèle Emmanuelli, Georges Pragier (dir.), Le traumatisme psychique, Paris, PUF, 2005, p. 144.
} 
Elle rappelle qu'un sujet n'advient à lui-même que s'il a pu « recueillir la voix de ses ascendants du fait même qu'il s'est éloigné de la portée captatrice de cette voix. C'est ce double mouvement qui rend communément possible la transmission ${ }^{51}$.

Elle est aussi sensible à la notion de travail dans la transmission issue de Benjamin qu'elle élargit dans le sens psychanalytique de travail psychique. Elle reprend dans cette perspective la fable de La Fontaine "Le laboureur et ses enfants » citée par Benjamin au début de son texte Expérience et pauvreté. Elle se demande avec lui : «Où les mourants prononcent-ils encore des paroles impérissables, qui se transmettent de génération en génération comme un anneau ancestral ? ${ }^{52}$. En effet, le travail est un «trésor» et il est menacé d'anéantissement par les violences d'État, comme tout ce qu'a produit la créativité de ce travail qui fait lien entre les hommes. Par ailleurs, hériter est aussi le fruit d'un travail comme elle le montre en évoquant ces vers de Goethe cités plusieurs fois par Freud: "Ce que tu as hérité de tes pères / acquiers-le afin de le posséder / ce qu'on n'utilise pas est un pesant fardeau ${ }^{53}$.

\section{Le don de récit comme don de mémoire}

La mémoire, qu'elle soit individuelle ou collective, joue un rôle essentiel dans la transmission. Dans Sur quelques thèmes baudelairiens, Benjamin s'appuie sur Bergson dans son argumentation :

[Bergson] considère la structure de la mémoire comme l'élément décisif pour la structure philosophique de l'expérience. Effectivement l'expérience appartient à l'ordre de la tradition, dans la vie collective comme dans la vie privée. Elle se constitue moins de données isolées, rigoureusement fixées par la mémoire, que de données accumulées, souvent inconscientes, qui se rassemblent en elle. ${ }^{54}$

Les données de l'expérience doivent être recherchées dans le temps: «Sans décryptage de l'expérience humaine passée, qu'on retrouve comme vie vécue dans les textes des ancêtres, les données de l'expérience du présent restent muettes $\gg{ }^{55}$.

\footnotetext{
${ }^{51}$ Ibid., p. 144.

${ }^{52}$ W. Benjamin, « Expérience et pauvreté », op. cit., p. 365.

${ }^{53} \mathrm{~J}$. Altounian, De la cure à l'écriture, op. cit., p. 96.

${ }^{54} \mathrm{~W}$. Benjamin, « Sur quelques thèmes baudelairiens », op. cit., p. 332.

55 Bernd Witte, «Paris - Lieu de mémoire », dans Topographies du souvenir : "Le Livre des passages » de Walter Benjamin, Paris, Presses Sorbonne Nouvelle, 2007, p. 6.
} 
L'auditeur ou le lecteur pour devenir conteur à son tour et continuer la transmission doit garder en mémoire les histoires reçues :

L'art de raconter les histoires est toujours l'art de reprendre celles qu'on a entendues, et celui-ci se perd dès que les histoires ne sont plus conservées en mémoire. Il se perd, parce qu'on ne file plus et qu'on ne tisse plus en les écoutant. Plus l'auditeur s'oublie lui-même, plus les mots qu'il entend s'inscrivent profondément en lui. ${ }^{56}$

Mais la réception des histoires doit être facilitée. Un «état de détente », « l'ennui », est nécessaire pour une bonne réception :

L'ennui est l'oiseau de rêve qui couve l'œuf de l'expérience. Au moindre bruit dans le feuillage, l'oiseau s'envole. Dans les villes - où il n'est plus d'activités qui soient intimement liées à l'ennui -, il ne trouve déjà plus aucun endroit pour faire son nid et, même à la campagne, il lui est de plus en plus difficile de s'établir. Ainsi se perd le don de prêter l'oreille. ${ }^{57}$

Pour employer un terme psychanalytique, un état de rêve ou de rêverie est nécessaire pour recevoir, contenir et penser comme Wilfred Bion $^{58}$ l'a montré, notamment dans son ouvrage Aux sources de l'expérience, titre éclairant, dans un après-coup, l'essai de Benjamin.

Dans la section XIII, Benjamin revient sur l'importance de la mémoire : «On s'est rarement rendu compte que la relation naïve de l'auditeur avec le conteur est dominée par l'envie de retenir l'histoire racontée pour pouvoir la raconter à son tour ${ }^{59}$. Le conteur suscite un intérêt et un plaisir immédiat chez l'auditeur mais aussi un plaisir plus différé quand l'auditeur racontera à son tour cette histoire. À l'origine, l'épopée contient à la fois le conte et le roman et « Mnémosyne, celle qui se remémore, était pour les Grecs la muse de l'épopée ${ }^{60}$. Le texte nomme «souvenir» cette mémoire épique. Deux modalités de la mémoire surgiront ensuite dans l'essai, selon le commentaire de Payot, une « mémoire distrayante» (récit) et une " mémoire éternisante » ou « remémoration » (roman). La mémoire du roman « est consacrée à un seul héros, un seul périple ou un seul combat» et serait ainsi identifiante.

\footnotetext{
${ }^{56}$ W. Benjamin, « Le conteur », op. cit., p. 126.

${ }^{57}$ Ibid., p. 126.

58 Wilfred R. Bion, Aux sources de l'expérience, traduit de l'anglais par François Robert, Paris, PUF, 2003 [1962].

${ }^{59}$ W. Benjamin, « Le conteur », op. cit., p. 134.

${ }^{60}$ Ibid., p. 134.
} 
La mémoire du récit s'intéresse, elle, à de nombreux événements et appelle à une multiplicité d'autres histoires. Elle serait ainsi réticulaire ${ }^{61}$. Quoi qu'il en soit, dans le passé mais aussi dans l'avenir, la chaîne de la tradition repose sur :

[...] la mémoire qui transmet de génération en génération les événements passés. Elle est la muse du genre épique dans son acceptation la plus large. Elle embrasse tous les sous-genres de l'épopée. Parmi ceux-ci figure au premier rang l'art incarné par le conteur. C'est la mémoire qui tisse le filet que forment en définitive toutes les histoires. Car celles-ci se raccordent toutes entre elles, comme les grands conteurs, particulièrement les Orientaux, se sont toujours plus à le souligner. En chacun d'eux vit une Schéhérazade, pour qui chaque épisode d'une histoire en évoque aussitôt une autre. ${ }^{62}$

Par l'utilisation à plusieurs reprises dans l'essai du terme don, Benjamin inscrit, même s'il ne fait pas référence à Marcel Mauss, le fait de raconter une histoire et de l'écouter ou de la lire dans le cycle du don et de l'échange et donc de fait dans la littérature anthropologique sur le don $^{63}$. Dans son célèbre Essai sur le don, Mauss, en 1923-1924, a individualisé les trois obligations du don (donner-recevoir-rendre), même si plus tard, pour Claude Lévi-Strauss, c'est l'échange qui constituera le phénomène primitif ${ }^{64}$. De nombreux auteurs ont travaillé depuis sur le don. Par exemple, Maurice Godelier reviendra sur la troisième obligation et, pour lui, il ne s'agit pas tant de rendre que de donner à son tour ${ }^{65}$.

Le conteur raconte une histoire au lecteur ou à l'auditeur qui va avoir envie de la raconter à son tour. Le conteur donne ainsi la possibilité au lecteur ou à l'auditeur de devenir conteur à son tour, de donner à son tour :

[L'auditeur] prête l'oreille aux histoires de telle façon que lui échoit naturellement le don de les raconter à son tour. Ainsi se noue le filet où repose le

\footnotetext{
${ }^{61}$ Daniel Payot, «Commentaire », dans Walter Benjamin, Le raconteur. A propos de l'œuvre de Nicolas Leskov, traduit de l'allemand par Sybille Muller, Strasbourg, Circé, 2014, p. 41-140.

${ }^{62}$ W. Benjamin, « Le conteur », op. cit., p. 137.

${ }^{63}$ Olivier Taïeb, Les histoires des toxicomanes. Récits et identités dans les addictions, Paris, PUF, 2011.

${ }^{64}$ Marcel Mauss, «Essai sur le don. Forme et raison de l'échange dans les sociétés archaïques », dans Idem, Sociologie et anthropologie, Paris, PUF, 1999 [1923-1924], p. 143-279.

Claude Lévi-Strauss, «Introduction à l'œuvre de Marcel Mauss », dans Marcel Mauss, Sociologie et anthropologie, Paris, PUF, 1999 [1950], p. IX-LI.

${ }^{65}$ Maurice Godelier, L'énigme du don, Paris, Fayard, 1996.
} 
don de raconter. Il se défait aujourd'hui par tous les bouts, après qu'il a été assemblé, voici plusieurs années, dans la sphère des plus anciennes formes d'artisanat. ${ }^{66}$

Dans la version de son essai qu'il a lui-même écrit en français, Benjamin insiste encore plus sur le don au début de la section XIII. La phrase «La mémoire est, entre toutes, la faculté la plus nécessaire à l'épopée ${ }^{67}$ disparaît et est remplacée par «La mémoire est le don épique par excellence ${ }^{68}$.

\section{L'histoire et la psychanalyse face à la chute du cours de l'expérience}

Malgré le constat fait tout au long de l'essai de l'éloignement de la forme traditionnelle du récit, Benjamin ne souhaite pas pour autant revenir en arrière. Il n'affirme pas la fin de la narration puisqu'elle subsiste évidemment dans le roman. Il évoque même un nouveau genre narratif: "Nous avons vu naître la short story qui s'est arrachée à la tradition orale $»^{69}$. Patricia Lavelle ${ }^{70}$ en souligne la similitude avec les kurze Prosa de Kafka que Benjamin a analysées, comme, par exemple, Le silence des Sirènes ${ }^{71}$. De nouvelles formes narratives sont donc toujours possibles et attestent ainsi que « la fonction narrative peut se métamorphoser mais non pas mourir. Car nous n'avons aucune idée de ce que serait une culture où l'on ne saurait plus ce que signifie raconter $\gg{ }^{72}$.

La psychanalyse peut aussi contribuer à revaloriser «le cours de l'expérience » et retisser les liens entre les morts et les vivants en favorisant des «rendez-vous» non mortifères avec les générations passées, pour reprendre le terme de Benjamin dans la Thèse II de Sur le concept d'histoire. Dans cet ultime texte écrit en 1940, Benjamin propose d'écrire l'histoire à partir du présent de l'historien. Pour lui, l'intelligibilité historique vient « de la rencontre entre un moment du

\footnotetext{
${ }^{66}$ W. Benjamin, « Le conteur », op. cit., p. 126.

${ }^{67}$ Ibid., p. 134.

${ }^{68}$ W. Benjamin, « Le Narrateur », op. cit., p. 283.

${ }^{69}$ W. Benjamin, « Le conteur », op. cit., p. 129.

${ }^{70}$ Patricia Lavelle, «Histoire, politique et narration: Benjamin lecteur de Kafka », Cités, 74, 2018, p. 77-90.

${ }^{71}$ Walter Benjamin. « Franz Kafka. Pour le dixième anniversaire de sa mort », traduit de l'allemand par Maurice de Gandillac revu par Pierre Rusch, dans Euvres II, Paris, Gallimard, coll. Folio Essais, 2000 [1934], p. 410-453.

${ }^{72}$ Paul Ricœur, Temps et récit. 2. La configuration dans le récit de fiction, Paris, Seuil, coll. Points Essais, 1991 [1984], p. 57.
} 
passé et un moment du présent, celui-là même où se situe l'historien $»^{73}$. De cette rencontre "naît une figure de pensée nouvelle, où le présent féconde le passé et réveille le sens oublié ou refoulé qu'il porte en lui, alors que le passé retrouve, au cœur du présent, une actualité nouvelle $\gg{ }^{74}$. Benjamin transpose ainsi la bidirectionnalité des processus psychiques avec leurs mouvements rétrogrédients et progrédients au temps de l'histoire conçu comme une expérience intérieure collective ${ }^{75}$. Il critique le temps continu accumulatif de l'idéologie du Progrès, construite par les Lumières et reprise ensuite par la pensée libérale et la pensée marxiste, pour défendre l'idée, empruntée au messianisme juif, d'une utopie surgissant au cœur même du présent, une utopie donnant une nouvelle chance à tout ce qui dans le passé a été manqué, oublié ou abandonné.

Dans l'idéologie du Progrès défendue par les historiens des vainqueurs, l'avenir est prévisible, le Même revient inlassablement et les injustices se perpétuent ${ }^{76}$. Face à ce danger, l'historien doit s'efforcer de sauver de l'oubli l'histoire des «vaincus», " des sans-noms » ${ }^{77}$ en revisitant le passé pour faire surgir quelque chose de nouveau. Tout ce qui dans le passé, tant personnel que collectif, a été manqué, a échoué ou n'a pas abouti, peut être ainsi réélaboré et remanié dans le présent, de même que tout ce qui a été oublié peut être rappelé à la mémoire. C'est, en effet, à partir des espoirs des générations passées que les rêves prennent forme pour Benjamin. L'utopie dépend ainsi de la mémoire :

C'est donc à nous de nous rendre compte que le passé réclame une rédemption dont peut-être une toute infime partie se trouve être placée en notre pouvoir. Il y a un rendez-vous mystérieux entre les générations défuntes et celle dont nous faisons partie nous-mêmes. Nous avons été attendus sur terre. Car il nous est dévolu à nous comme à chaque équipe humaine qui nous précéda, une parcelle du pouvoir messianique. Le passé la réclame, a droit sur elle. Pas moyen d'éluder sa sommation. $^{78}$

\footnotetext{
73 Stéphane Mosès, L'Ange de l'Histoire. Rosenzweig, Benjamin, Scholem, Paris, Gallimard, coll. Folio essais, 2006 [1992], p. 223.

${ }^{74}$ Ibid., p. 224.

75 Olivier Taïeb, «Enfance berlinoise vers 1900 de Walter Benjamin : une enfance remémorée selon le concept de l'après-coup de Freud », Anthropology and Materialism, 4, 2019.

${ }^{76}$ Michaël Löwy, Walter Benjamin : Avertissement d'incendie. Une lecture des Thèses "Sur le concept d'histoire », Paris, L'éclat, 2014.

${ }^{77}$ W. Benjamin, « Sur le concept d'histoire », op. cit., p. 454.

${ }^{78}$ Ibid., p. 433-434.
} 
Si le présent se tourne vers le passé, ce n'est pas pour l'interpréter ou pour y chercher ce qui l'intéresse, mais c'est d'abord parce que celuici l'interpelle. Le présent ne peut pas se soustraire à cet appel à l'aide des « vaincus ${ }^{79}$ de l'histoire ${ }^{80}$.

La remémoration ne se contente donc pas seulement d'évoquer un moment du passé, elle vise à le transformer parce qu'il est possible d'intervenir pour en changer dans l'après-coup sa signification. Dans ce processus, il s'agit de déchiffrer les traces que le passé a laissées : «Le passé a laissé de lui-même des images comparables à celles que la lumière imprime sur une plaque photosensible. Seul l'avenir possède des révélateurs assez actifs pour fouiller parfaitement de tels clichés ${ }^{81}$.

Benjamin propose ainsi une histoire où chaque moment du passé peut être réactualisé, rejoué dans d'autres conditions, sur une nouvelle scène. Le messianisme n'est pas conçu comme l'attente d'une apothéose qui se produirait au terme d'un temps linéaire et continu mais comme la possibilité donnée à chaque moment du temps, de l'avènement du nouveau.

La crise de la transmission n'est donc pas inéluctable. Il est possible d'en envisager une issue face à la répétition traumatique. L'historien dans une perspective collective et le psychanalyste dans une perspective individuelle et familiale peuvent ainsi (re)valoriser « le cours de l'expérience » en exerçant leur « parcelle de pouvoir messianique ». Dans le même mouvement, un des enseignements «cachés» de Benjamin est, peut-être, de «sauver de l'oubli» l'ambition révolutionnaire de la psychanalyse et de révéler, dans l'après-coup, sa dimension politique individuelle et collective.

\footnotetext{
${ }^{79}$ Benjamin se considère comme un « vaincu » de l'histoire : « Notre génération à nous est payée pour le savoir, puisque la seule image qu'elle va laisser est celle d'une génération vaincue. Ce sera là son legs à ceux qui viennent » (Ibid., p. 440).

${ }^{80}$ Philippe Simay, « Reconstruire la tradition. L'anthropologie philosophique de Walter Benjamin », Cahiers d'anthropologie sociale, ${ }^{\circ} 4$, 2008, p. 87-98.

${ }^{81}$ W. Benjamin, « Sur le concept d'histoire », op. cit., p. 452.
} 